\title{
Lagrange and the foundations of analysis
}

\author{
Sandro Caparrini
}

Published online: 22 May 2014

(c) Centro P.RI.ST.EM, Università Commerciale Luigi Bocconi 2014

\begin{abstract}
A brief description of the historical development of the ideas that led Lagrange, in 1797, to refute the concept of limit and base differential calculus on power series expansions.
\end{abstract}

Keywords Lagrange - History of analysis - 18th-century mathematics · Series expansions · Limit - Differential calculus

The first methods proposed by mathematicians for the foundations of infinitesimal calculus were insufficient, sometimes absurd. Leibniz, in 1684, worked with quantities small enough to be considered null. Newton, in 1687, imagined points and lines in motion. Euler, in 1755, believed that the ratio $0 / 0$ might be a quantity other than zero. D'Alembert, in 1765, asserted that infinitesimal calculus was based on the concept of limit, but did not go further into this intuition. Cauchy, in 1821, worked systematically with limits, but those limits were neither well formalised nor based on any theory of real numbers. It would not be until the end of the 1800s that a solid basis for real analysis would be found.

In this gallery of brilliant yet problematic ideas, the method of "analytical functions" published by Lagrange in 1797 [7] does not make a bad impression. It is true that today it would be unacceptable, but we must remember that from that time more than two centuries of tumultuous progress in mathematics have gone by. If we place ourselves in the proper historical perspective, Lagrange's theory appears simple and elegant $[2,3,4,5]$.

S. Caparrini $(\bowtie)$

Dipartimento di Matematica, Università di Torino, Via Carlo

Alberto, 10, 10123 Turin, Italy

e-mail: sandro.caparrini@unito.it
Lagrange constructed his method a little at a time. He took the first step when, barely 19 years old, he was assigned to teach a course in infinitesimal calculus at the School of Artillery in Turin. In a letter to Euler dated 24 November 1759, he writes of having found the vray metaphisique (that is, the true foundations) of differential calculus.

What this metaphisique was we can know from a copy of the notes of his lessons, written between 1756 and 1759, which have come down to us [1]. In essence, Lagrange develops the first elements of the theory of finite differences, then obtains the differential expressions as the limits of the ratios of these differences. That is, he takes from Leibniz the idea of differential calculus as a calculus of extremely small finite differences, and applies to the formulas thus obtained the method of "last ratios" expounded by Newton in the Principia of 1687. This procedure resembles that followed by Euler in his Institutiones calculi differentialis of 1755, which, however, Lagrange did not yet know at the time.

It might seem that he was moving in the right direction. Instead Lagrange later abandoned this route. Why? The fact is that limits seemed as uncertain as infinitesimals. In fact, it was not clear what the ratio of two quantities was when both became equal to zero. Newton had tried to explain it, but he limited himself to a few examples. Without the notorious epsilon and delta, so hated by our students, the concept of limit remained outside of rigorous mathematics.

The germ of the theory of analytical functions is found in a 1774 memoir entitled "Sur une nouvelle espèce de calcul relatif à la différentiation \& à l'intégration des quantités variables" [6]. This "new calculus", in modern terminology, is a sketch of the theory of differential operators. Since the work is based on power series 
expansion, the first part is dedicated to a new proof of Taylor's formula. Lagrange supposes that each function can be expanded in a series of the form $f(x+i)=$ $f(x)+p(x) i+q(x) i^{2}+r(x) i^{3}+\cdots$ etc., where $x$ and $i$ are arbitrary real quantities. The idea underlying the proof is that of expanding separately the two expressions $f((x+o)+i)$ and $f(x+(o+i))$, and of then setting them equal to each other term by term.

At this point Lagrange introduces a new notation: the coefficient $p(x)$, which "derives" from $f(x)$, is denoted with $f^{\prime}(x)$; in the same way, the coefficient of the first power in the expansion of $f^{\prime}(x)$ is denoted $f^{\prime \prime}(x)$, and so on. This is the first appearance of "derived functions".

Lagrange's proof presents several advantages with respect to those known during that period. It presumes no knowledge of differential calculus; it does not use infinitesimals; and the derivatives appear in a natural way. Above all, Lagrange's derivatives are functions, not ratios of differentials.

Lagrange saw in this theorem the key for a rigorous foundation of analysis.

Le Calcul différentiel, considéré dans toute généralité, consiste à trouver directement e par des procédés simples et faciles, les fonctions $\mathrm{p}, \mathrm{p}^{\prime}, \mathrm{p}^{\prime \prime} \ldots, \mathrm{q}, \mathrm{q}^{\prime}$, $\mathrm{q}^{\prime \prime} \ldots, \mathrm{r}, \mathrm{r}^{\prime}, \mathrm{r}^{\prime \prime} \ldots$ dérivés de la fonction $\mathrm{u}$, et le Calcul intégral consiste à retrouver la fonction u par le moyen de ces dernières fonctions.

Cette notion des Calculs différentiel et intégral me parait la plus claire et la plus simple qu'on ait encore donnée; elle est, comme on voit, indépendante de toute métaphysique et de toute théorie des quantités infiniment petit ou évanouissantes [9, vol. III, p. 443]. (Differential calculus, considered in all generality, consists in finding directly and by means of simple and easy procedures the functions $p, p^{\prime}, p^{\prime \prime} \ldots, q, q^{\prime}$, $q^{\prime \prime} \ldots, r, r^{\prime}, r^{\prime \prime} \ldots$ derived from the function $u$; and integral calculus consists in finding anew the function $u$ by means of these last functions.

This notion of differential and integral calculus seems to me the clearest and simplest that has been give so far; it is, as one sees, independent of any metaphysics and of any theory of quantities that are infinitely small or evanescent).

It would be a quarter of a century before Lagrange elaborated this idea. Yet another time, he was driven by the requirements of teaching. In 1795 he was assigned to teach infinitesimal calculus at the École centrale des travaux publics (later the École Polytechnique). Two texts were born of this course: the Théorie des fonctions analytiques [7] and the Leçons sur les fonctions analytiques [8]. Lagrange hurriedly wrote the Théorie to provide a reference work for the students, adding to pure analysis the applications to differential geometry and mechanics. The Théorie is thus Lagrange's version of a complete treatise of analysis. Later were published the Leçons, which correspond to what was expounded in the course. The two texts overlap and complete each other.

As in all the treatises by Lagrange, the Théorie and the Leçons contain interesting historical discussions, but no illustrations. The form of the exposition recalls that of a treatise of algebra in which to the usual algebraic operations have been added the operation of differentiation and its inverse, integration. Historians have named this methodology "differential algebra".

The memoir of 1774 [6] postulated the possibility of a power series expansion; it was now necessary to prove it. In modern texts it is necessary to impose very stringent conditions of regularity to obtain Taylor series. Some mathematicians have thus been surprised to see that Lagrange presumes to expand any function whatsoever. In reality, Lagrange considers classes of functions that are much more restricted than those of today. In the first place, he uses a definition of function, taken from Euler, that is very different from our abstract correspondences between sets; his functions are formulas and can assume more than one value. In the second place, he works only with algebraic and elementary transcendental functions.

With these limitations, it is possible to come close to powerful results by means of simple considerations. Lagrange proves immediately that it is not possible to obtain expansions with fractional exponents, of the form $f(x+i)=f(x)+i p+i^{2} q+i^{3} r+\cdots+u i^{m / n}+\cdots$, etc.

In fact, the possible presence of the radical $\sqrt{[n]} i^{m / n}$, which is a multivalued function, would make the series assume more values than the original function. In a similar fashion, he proves that it is not possible for the exponents to assume negative values: for $i=0$ there would be infinite $f(x)$ for any arbitrary value of $x$, which is absurd.

Now the possibility of series expansion can be proved. Lagrange divides $f(x+i)$ into two parts: one that depends on $i$, and one that does not. And since, all told, he is working with algebraic functions, and given that the powers of $i$ are positive integers, it is possible with a few passages to factor $i$; thus $f(x+i)=f(x)+i P(x, i)$, where the function $P(x, i)$ remains finite if $i=0$. Likewise, $P(x, i)$ is broken down into the sum $p(x)+i Q(x, i)$. Iterating the procedure, he obtains the series expansion of $f(x)$. Note that this process bears a certain resemblance to the hypothesis, in modern terms, of infinite differentiability.

Lagrange knows that there can be singular points at which the Taylor series does not exist, and carefully examines the question in the fifth chapter of the Théorie. But this concerns the singularity of algebraic functions: 
essentially cusps and multiple points. We are far from the impossible-to-visualise monstrosities of modern analysis.

At the heart of the theory is found the remainder theorem: arresting the expansion, say, at the second term, we can account for the remaining terms of the series by means of the formula

$f(x)=f(0)+x f^{\prime}(0)+\frac{x^{2}}{2} f^{\prime \prime}(0)+\frac{x^{3}}{2 \times 3} f^{\prime \prime \prime}(u)$

where $u$ is an intermediate value between 0 and $x$. Though Lagrange maintained a façade of modesty, he was aware of the importance of his result.

We must however grasp the significance of this formula. The mathematicians of the 1700s knew intuitively that a series must be completed by a remainder. The remainder of the geometric series was well known, and in 1754 d'Alembert had found a remainder of a complicated form that was generally valid. But this was a question of numerical calculations, which did not concern the theoretical validity of the expansion. Series were used to obtain numerical values, for example, in astronomy, and it was thus necessary to be able to evaluate the order of magnitude of the error. Lagrange's theorem made a simple expression for these calculations available.

Looking at the proof of the remainder theorem, one is struck by the systematic use of inequalities to bound suitably certain quantities. The infinitesimal calculus of Euler and d'Alembert did not proceed in this way; we almost seem to see a text of the century that was just about to begin. There is no doubt that the mathematicians of the next generation were influenced by this way of proceeding. It is an almost indiscernible step forward, far from Kuhn's scientific revolutions. But it is a step forward.

What remains today of Lagrange's analytical functions? A lot. First of all, the notation and terminology. Our $f^{\prime}(x)$ comes directly from the Théorie; our derivative is Lagrange's fonction dérivée. A modern reader, who is perplexed by almost every line of Newton and Leibniz, can read the Théorie and the Leçons better than certain novels.

Passing to the mathematical results, the influence of analytical functions is equally pervasive. After Euler and Lagrange, no one doubted that the foundations of analysis had to be purely analytical. The remainder theorem became central to the theory. The derived function definitively substituted the ratios of differentials.

The defects of the theory were influential as well. In a treatment that was intended to be completely analytical, geometry is invoked in essential passages. It seemed obvious, for example, that a curve can pass from negative to positive values only by cutting the Cartesian axis at some point. It would take mathematicians almost a century to correct these defects.
All in all, the principal problem was that Lagrange's analytical functions looked to the past rather than to the future. Within just a few years after the publication of the Théorie, a mass of new and unexpected results accumulated in the pages of journals of mathematics. In order to take them into account, the young Cauchy reconstructed analysis from the foundations; his Analyse algébrique of 1821 owed a debt to Lagrange that is already shown in the title. Then, slowly, even the Analyse was superseded. Today both Lagrange and Cauchy are often cited but little read, and it remains to the historian to recount what we owe to each of them.

(Translated from the Italian by Kim Williams)

\section{References}

1. Borgato, M., Pepe, T.: L. Lagrange a Torino (1750-1759) e le sue lezioni inedite nelle R. Scuole di Artiglieria. Bollettino di storia delle scienze matematiche 7, 3-43 (1987)

2. Bottazzini, U.: Il calcolo sublime: storia dell'analisi matematica da Euler a Weierstrass. Turin, Boringhieri (1981)

3. Dugac, P.: Histoire de l'analyse: autour de la notion de limite et de ses voisinages. Vuibert, Paris (2003)

4. Fraser, C.G.: Calculus and Analytical Mechanics in the Age of Enlightenment. Aldershot, Variorum (1997)

5. Fraser, C.G., Lagrange, J.L.: Théorie des fonctions analytiques, first edition (1797). In: Grattan-Guinness, I. (ed.) Landmark Writings in Western Mathematics: 1640-1940, pp. 258-276. Elsevier, Amsterdam (2005)

6. Lagrange, J.L.: Sur une nouvelle espèce de calcul relatif à la differentiation \& à l'intégration des quantités variables. Nouv. Mém. Berlin, année 1772, pp. 185-221 (1774). Rpt. (Lagrange, Oeuvres III, pp. 441-476)

7. Lagrange, J.L.: Théorie des fonctions analytiques contenant les principes du calcul différentiel, dégagés de toute considération d'infiniment petits ou d'évanouissans, de limites ou de fluxions, et réduits à l'analyse algébrique des quantités finies. Imprimerie de la République, Paris (1797). Rpt. (Lagrange, Oeuvres IX)

8. Lagrange, J.L.: Leçons sur le calcul des fonctions. Imprimerie du Cercle social, Paris (1801). Rpt. (Lagrange, Oeuvres X)

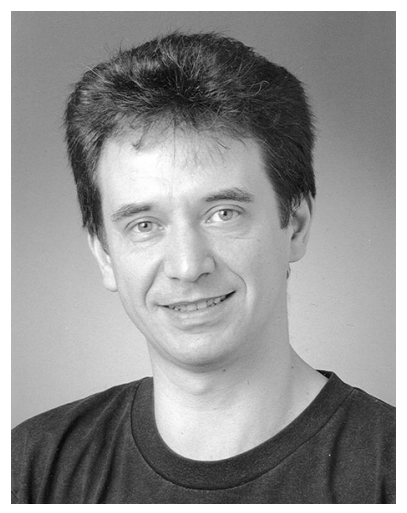

Sandro Caparrini holds degrees in Physics and in Mathematics and a Ph.D. in Mathematics from the University of Turin. His research interests are mainly focused on the history of the interaction between mathematics and mechanics from 1750 onward. He has held postdoctoral positions at the Dibner Institute (Harvard and MIT), at the Cohn Institute (Tel Aviv), at the Institute for the History and Philosophy of Science and Technology (Toronto) and at the Department of Mathematics of the University of Lille (France). In 2004 he was awarded the Slade Prize from the British Society for the History of Science. 